\title{
A Espectrometria de Massa de Ressonância Ciclotrónica de Iões com Transformada de Fourier
}

\author{
J O A Q U IM M R ÇA L O *
}

\begin{abstract}
A espectrometria de massa de ressonância ciclotrónica de iões com transformada de Fourier (FT-ICR/MS) é uma técnica de análise de massas que consiste no confinamento e detecção de iões numa região do espaço definida por um campo magnético e um campo eléctrico homogéneos, $\mathrm{e}$ que encontra aplicação nos domínios da química analítica e da química-física.
\end{abstract}

\section{INTRODUÇÃO}

A espectrometria de massa é uma técnica instrumental bastante poderosa ao dispor dos químicos e outros cientistas para o estudo de problemas fundamentais ou aplicados em áreas tão diversas como, por exemplo, a bioquímica, a catálise, a química do ambiente, a ciência dos materiais, a geoquímica, a física das partículas e a cosmologia. De entre os vários tipos de técnicas de análise de massas existentes, uma das mais versáteis e com uma utilização em franca expansão nos últimos anos é, sem dúvida, a espectrometria de massa de ressonância ciclotrónica de iões com transformada de Fourier (que abreviaremos por FT-ICR/MS, optando por manter as siglas derivadas das designações em língua inglesa por serem as que irão ser encontradas na literatura da especialidade). Esta técnica tem como características principais um elevado poder de resolução de massas e uma elevada exactidão na sua determinação, a detecção simultânea de todos os iões com um grande intervalo de massas, a possibilidade de armazenar os iões por períodos longos e de os manipular, de efectuar medidas múltiplas dos mesmos iões, de realizar espectrometria de massa "tandem" (MS/MS/...) e a compatibilidade com uma grande variedade de técnicas de ionização. Nesta descrição da espectrometria de massa FT-ICR serão essencialmente abordadas as características da técnica e será feita apenas uma breve referência às aplicações no âmbito da química, indicando-se no final diversa bibliografia que melhor poderá ilustrar essas aplicações.

\section{FT-ICR/MS: PRINCÍPIOS}

A espectrometria de massa FTICR é uma técnica de análise de massas que emprega um campo magnético e um campo eléctrico estáticos para confinar os iões numa região finita do espaço e aí os detectar. Toda a experiência de FT-ICR/MS ocorre numa armadilha de iões ("ion trap"). geralmente de geometria cúbica ou cilíndrica, que é mantida sob vácuo ultra-elevado no centro do campo magnético, habitualmente produzido por um magnete supercondutor.

A armadilha ou célula de confinamento de iões ("trapped ion cell", Figura 1) é formada por três pares de pratos em oposição que têm funções específicas de confinamento ("trapping"), excitação e deteç̧ão dos iões a serem analisados. Convencionalmente, em coordenadas cartesianas, $z$ coincide com a direcção do campo magnético e é perpendicular aos pratos de confinamento, enquanto que $x$ é perpendicular aos pratos de excitação (ou transmissão) e $y$ aos de deteç̧ão (ou recepção). A célula está colocada na zona homogénea do campo magnético de tal forma que este confina cada ião radialmente, obrigando-o a descrever um movimento de ciclotrão (ver adiante) com uma frequência que é função da respectiva razão massa/carga, enquanto que o campo eléctrico gerado por um potencial electrostático aplicado aos pratos de confinamento mantém o ião num fosso de potencial na direcção de $z$. A célula de ICR representada na Figura 1 possui geometria cúbica. Esta é a geometria mais comum mas não é a única utilizada em espectrómetros de massa FT-ICR (ver adiante a Figura 4).

Numa experiência de FT-ICR típica, os iões presentes na célula, previamente gerados por um método de ionização adequado, são sujeitos a um campo eléctrico oscilante de radiofrequências transmitido através dos pratos de excitação; quando as radiofrequências entram em ressonância com as frequências de ciclotrão dos iões, há absorção de energia que força os iões a deslocarem-se para órbitas de maior raio, aproximando-se dos pratos de detecção, nos quais induzem uma corrente imagem que é uma sobreposição das frequências de ciclotrão dos diferentes iões sujeitos a excitação, com amplitudes que são proporcionais ao número de iões. O sinal transiente detectado é convertido numa voltagem, digitalizado e sujeito a uma transformada de Fourier. a qual revela as frequências e intensidades que são depois convertidas em massas e abundâncias iónicas.

Aspectos históricos. A origem da espectrometria de massa FT-ICR remonta à descrição feita em 1930 por E. O. Lawrence, inventor do acelerador de ciclotrão, do princípio fundamental da ressonância ciclotrónica de iões: partículas carregadas são forçadas a mover-se em órbitas circulares com um período de revolução constante quando situadas num campo magnético uniforme, e podem ser aceleradas por exposição a um campo eléctrico oscilante com uma frequência igual à frequência de ciclotrão das partículas. Ainda nos anos 30, Penning projectou a primeira armadilha ("trap") para partículas carregadas, utilizando uma combinação de campo eléctrico e magnético estáticos para confinar electrões.

O princípio de ICR foi aplicado pela primeira vez à espectrometria de massa em 1949 por Hipple, Sommer e Thomas, que desenvolveram um

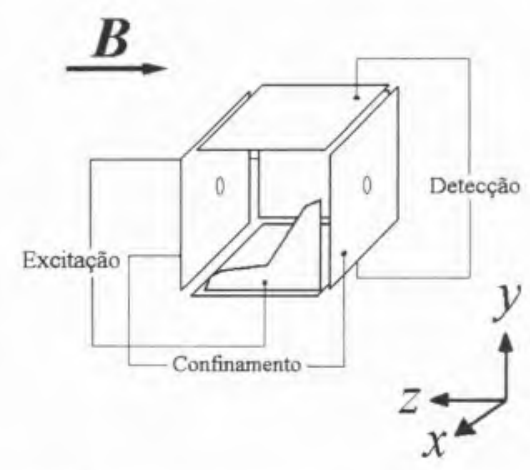

Fig. 1 - Representação esquemática de uma célula de ICR cúbica. 
instrumento designado por omegatrão no qual os iões, em presença de um magnete permanente, eram acelerados radialmente na respectiva frequência ciclotrónica por um campo eléctrico alterno e detectados num colector fixo; devido ao reduzido poder de resolução e pequeno intervalo de massas, o aparelho teve uma utilização bastante limitada. Alguns anos mais tarde, Wobschall e colaboradores introduziram um novo tipo de detector que aumentou significativamente a sensibilidade dos aparelhos de ICR; utilizaram um campo magnético variável proveniente de um electromagnete, com o qual provocavam a ressonância sequencial dos diferentes iões. Llewellyn e o grupo de Baldeschwieler desenvolveram, entretanto, uma "célula" de iões ("drift cell") dividida em três regiões, fonte, analisador e colector, ao longo da qual os iões se moviam à deriva ("drift") por acção de um campo eléctrico estático perpendicular ao campo magnético; a principal vantagem desta geometria era a separação das regiões de ionização e de ressonância. Esta célula de iões e o detector introduzido por Wobschall foram constituintes essenciais dos primeiros espectrómetros de ICR comerciais, que foram utilizados principalmente para o estudo de reacções ião-molécula em fase gasosa.

Um dos avanços instrumentais mais importantes da técnica de ICR foi devido a McIver, com o desenvolvimento em 1970 da "célula de confinamento de iões" ou "armadilha de iões" ("trapped ion cell" ou "ion trap"), em que os iões, formados, armazenados e detectados num único compartimento, podiam ser conservados durante períodos de tempo duas ordens de grandeza superiores aos observados na "drift cell", melhorando assim a sensibilidade, $o$ intervalo dinâmico e a resolução de massas. A evolução da espectrometria de ICR para a sua forma moderna de FT-ICR culminou com a introdução por Comisarow e Marshall em 1974 de um novo sistema de detecção que, através da aplicação de uma transformação de Fourier, permitia obter um espectro de massa completo no tempo anteriormente necessário para determinar um único pico; a utilização de um campo magnético estático e a separação efectiva entre a excitação e a detecção conduziu a grandes melhorias de eficiência, particularmente ao nível da resolução.

Aspectos fundamentais. Um ião de massa $m$, carga $q$ e velocidade $\boldsymbol{v}$, quando em presença de um campo magnético $\boldsymbol{B}$ (na direcção de z), fica sujeito a uma força $F$ de Lorentz (equação 1) que obriga o ião a mover-se numa órbita circular, ou seja, a executar um movimento de ciclotrão, no plano $x y$, tal como se mostra na Figura 2 para o caso de um ião com carga positiva.

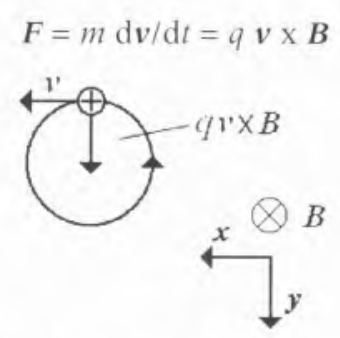

Fig. 2 - Representação do movimento de ciclotrão de um ião positivo devido à presença de um campo magnético na direcção perpendicular ao plano do papel.

Se $v_{x y}$ for a velocidade do ião no plano $x y, r$ o raio da órbita ciclotrónica e $B$ a intensidade do campo magnético (designação de $B$ habitualmente encontrada na bibliografia, correctamente, densidade de fluxo magnético), a equação 1 conduz à seguinte relação

$$
m v_{x y} 2 / r=q v_{x y} B
$$

Uma vez que a velocidade angular do ião, $\omega$, se define como $\omega=v_{x y} / r$, a equação 2 pode ser reescrita para dar a equação fundamental de ciclotrão

$$
\omega_{\mathrm{C}}=q B / m
$$

onde $\omega_{\mathrm{C}}$ designa a frequência de ciclotrão do ião, em rad $\mathrm{s}^{-1}, \operatorname{com} q \mathrm{em}$ Coulomb, $B$ em Tesla e $m$ em kg.

A equação de ciclotrão mostra, em primeiro lugar, que iões com uma dada razão massa/carga têm a mesma frequência de ciclotrão, independentemente da respectiva velocidade inicial, o que faz com que, contrariamente a outras técnicas de espectrometria de massa, não seja necessário "focar" a energia de translação dos iões para se proceder a uma determinação precisa das respectivas massas.

A equação 2 pode ser rearranjada para dar o raio da órbita ciclotrónica de um ião

$$
r=m v_{x y} / q B
$$

equação que pode ser escrita de outra forma considerando um ião com energia térmica, para o qual $v_{x y}=(2 k T / m)^{1 / 2}$, em que $k$ é a constante de Boltzmann e $T$ é a temperatura (em Kelvin),

$$
r=\left(2 m k T / q^{2} B^{2}\right)^{1 / 2}
$$

Através da equação 5 podemos verificar, por exemplo, que, num campo magnético de 3 Tesla e à temperatura ambiente, um ião de carga unitária e massa 100 u (unidade de massa atómica unificada, também designada por Dalton, Da) tem um raio orbital de ciclotrão de cerca de 0,08 mm, e até um ião de massa 10000 u tem um raio de apenas cerca de 0,8 $\mathrm{mm}$, valores que mostram bem a capacidade de confinamento do campo magnético que torna experimentalmente realizável a espectrometria de massa FT-ICR, inclusive para moléculas de massa bastante elevada.

Embora o campo magnético restrinja o movimento dos iões radialmente, estes são livres de se mover na direcção de $z$, paralela ao campo magnético, pelo que se torna necessário introduzir um campo eléctrico que restrinja o movimento dos iões axialmente. Este campo eléctrico é normalmente produzido por aplicação de uma pequena voltagem nos pratos de confinamento, que cria um fosso de potencial na direcção de $z$, dando origem a dois novos tipos de movimento dos iões, de oscilação axial e de magnetrão, além de introduzir uma perturbação no movimento de ciclotrão. 
Na região central da célula de ICR, o potencial aplicado aos pratos de confinamento é aproximadamente quadrupolar, ficando os iões sujeitos a um movimento de oscilação axial que corresponde a uma oscilação harmónica entre os dois pratos, ao longo da direcção de $z$, com uma frequência $\omega_{\mathrm{T}}$ que é dada por

$$
\omega_{\mathrm{T}}=\left(4 \alpha q V_{\mathrm{T}} / m a^{2}\right)^{1 / 2}
$$

em que $a$ é a distância entre os pratos de confinamento, $\alpha$ é uma constante que depende da forma da célula e $V_{\mathrm{T}}$ é a voltagem aplicada.

O campo eléctrico, para além da componente axial, que conduz ao movimento de oscilação axial, tem uma componente radial que produz uma força que se opõe à força magnética de Lorentz (ver Figura 2), isto é, a introdução do potencial de confinamento efectivamente reduz a intensidade do campo magnético.

Na presença de um campo magnético, $\boldsymbol{B}$, e de um campo eléctrico, $E$, a equação de Lorentz (equação 1) tem a forma

$$
m \mathrm{~d} v / \mathrm{d} t=q \boldsymbol{v} \times \boldsymbol{B}+q \boldsymbol{E}
$$

que, como anteriormente, para o plano $x y$ conduz à seguinte equação

$$
m \omega^{2} r=q \omega r B-q r E
$$

Resolvendo em ordem a $\omega$, obtêm-se duas frequências características, a de ciclotrão reduzida, $\omega_{\mathrm{R}}$, $\mathrm{e}$ a de magnetrão, $\omega_{\mathrm{M}}$ :

$$
\begin{aligned}
& \omega_{\mathrm{R}}=\left[q B+\left(q^{2} B^{2}-4 m q E\right)^{1 / 2}\right] / 2 m \\
& \omega_{\mathrm{M}}=\left[q B-\left(q^{2} B^{2}-4 m q E\right)^{1 / 2}\right] / 2 m
\end{aligned}
$$

Para um potencial eléctrico quadrupolar

$$
E=2 \alpha V_{\mathrm{T}} / a^{2}
$$

pelo que, substituindo esta relação nas equações 9 e 10 , utilizando a equação fundamental de ciclotrão (equação 3), a equação que traduz a frequência de oscilação axial (equação 6) e rearranjando, obtém-se
$\omega_{\mathrm{R}}=\left(\omega_{\mathrm{C}} / 2\right)+\left[\left(\omega_{\mathrm{C}} / 2\right)^{2}-\left(\omega_{\mathrm{T}}^{2 / 2}\right)\right]^{1 / 2}$

$\omega_{\mathrm{M}}=\left(\omega_{\mathrm{C}} / 2\right)-\left[\left(\omega_{\mathrm{C}} / 2\right)^{2}-\left(\omega_{\mathrm{T}}^{2} / 2\right)\right]^{1 / 2}$

sendo fácil verificar (ver equação 6) que na ausência de campo eléctrico $\left(V_{\mathrm{T}}=0\right) \omega_{\mathrm{T}}=0 \mathrm{e}$, consequentemente, $\omega_{R}=\omega_{C}$ e $\omega_{M}=0$. Das equações 12 e 13 resultam também as relações $\omega_{\mathrm{C}}=\omega_{\mathrm{R}}+\omega_{\mathrm{M}}$ e $\omega_{\mathrm{C}}^{2}=\omega_{\mathrm{R}}^{2}+\omega_{\mathrm{M}}^{2}+$ $\omega_{\mathrm{T}}^{2}$.

O movimento de magnetrão corresponde a uma precessão dos iões no plano $x y$, em torno de linhas equipotenciais, e na célula de ICR os iões descrevem trajectórias que combinam os movimentos de ciclotrão, de magnetrão e de oscilação axial. Na Figura 3 encontra-se uma representação esquemática dos movimentos dos iões numa célula de ICR.

As equações que definem as frequências dos três movimentos mostram que a frequência de ciclotrão depende fortemente da razão massa/carga, pois varia com $(m / q)^{-1}$, enquanto que a frequência de oscilação axial tem uma dependência menos acentuada com $(\mathrm{m} / \mathrm{q})^{-1 / 2}$; por sua vez, a frequência de magnetrão é praticamente independente daquela razão, podendo ser aproximada pela expressão $\omega_{\mathrm{M}} \approx 2 \alpha V_{\mathrm{T}} / a^{2} B$.

Para exemplificar esta dependência em relação à razão massa-/carga, bem como a grandeza relativa das três frequências (utilizando agora $v=\omega / 2 \pi)$, considere-se a situação típica de uma célula de ICR cúbica $(\alpha=1,386) \operatorname{com} a=2,54 \mathrm{~cm}, B=3 \mathrm{~T}$ e $V_{\mathrm{T}}=1 \mathrm{~V}$ : um ião de massa $100 \mathrm{Da}$ e carga unitária terá uma frequência de ciclotrão $v_{\mathrm{R}}=460,43 \mathrm{kHz}$, uma frequência de oscilação axial $v_{\mathrm{T}}=14,496$ $\mathrm{kHz}$ e uma frequência de magnetrão $v_{\mathrm{M}}=228 \mathrm{~Hz}$, enquanto que para um ião com a mesma carga e massa 1000 Da, $v_{\mathrm{R}}=45,837 \mathrm{kHz}, v_{\mathrm{T}}=4,584 \mathrm{kHz}$ e $v_{M}=229 \mathrm{~Hz}$.

Uma consequência importante da presença do campo eléctrico é o facto de existir uma massa a partir da qual a trajectória de um ião na célula de ICR deixa de ser estável: para uma massa suficientemente grande, a expressão sujeita a raiz quadrada na equação 9 torna-se negativa, o que corresponde, fisicamente, a uma situação em que o campo magnético não é suficientemente forte para se opor à componente radial do campo eléctrico, entrando os iões num movimento em espiral para o exterior ilimitado. Esta "massa crítica", $m_{c}$, pode ser facilmente encontrada considerando a equação 12 e a condição $\left(\omega_{\mathrm{C}} / 2\right)^{2}-\left(\omega_{\mathrm{T}}^{2 / 2}\right)=0$, que conduz a

$$
m_{\mathrm{C}}=q a^{2} B^{2} / 8 \alpha V_{\mathrm{T}}
$$

Exemplificando para a célula de ICR e as condições experimentais anteriormente referidas, obtém-se $m_{\mathrm{c}}=$ 50492 u.

O movimento orbital descrito pelos iões na célula de ICR não origina, por si só, qualquer sinal eléctrico observável nos pratos de detecção. Após a sua formação ou introdução na célula, iões com a mesma razão massa/carga e velocidade inicial começam o seu movimento aleatoria-

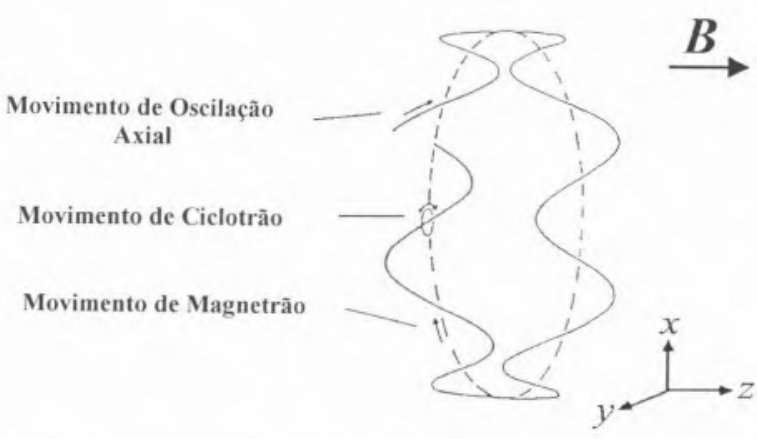

Fig. 3 - Representação esquemática dos três tipos de movimento descritos por um ião numa célula de ICR. 
mente no espaço, ou seja, não existe coerência de fase. Qualquer carga induzida por um ião num dos pratos de detecção será compensada, em média, por uma carga igual induzida no outro prato de detecção por um ião que, embora na mesma órbita, tenha uma fase que o coloque a $180^{\circ}$ do primeiro.

Com a aplicação de um campo eléctrico oscilante de radiofrequências aos pratos de excitação, iões com frequências de ciclotrão correspondentes à frequência do campo aplicado irão entrar em ressonância, absorvendo energia, movendo-se continuamente para órbitas maiores (seguindo uma trajectória em espiral) e adquirindo coerência de fase. Assim, iões com a mesma razão massa/carga passarão a mover-se agrupados no que é frequentemente referido como um "pacote" de iões ("ion packet").

Como vimos anteriormente, a frequência de magnetrão é praticamente independente de $\mathrm{m} / \mathrm{q}$ (excepto para valores muito elevados desta grandeza), enquanto que se observa uma dependência acentuada no caso da frequência de ciclotrão, o que a torna uma medida ideal da razão massa/carga de um ião. A frequência de oscilação axial apresenta tipicamente valores bastante inferiores à frequência de ciclotrão, pelo que, embora experimentalmente possível, não é normalmente utilizada para a detecção de iões em FT-ICR.

Considerando que a energia absorvida (por um ião inicialmente em repouso) durante o tempo de excitação, $t$, é convertida em energia cinética, chega-se à seguinte relação

$E_{\text {cin }}=q^{2} E_{0}^{2} t^{2} / 8 m$

em que $E_{0}$ é a intensidade do campo eléctrico de radiofrequências. Como, pelas equações 2 e 3 ,

$E_{\mathrm{cin}}=q^{2} r^{2} B^{2} / 2 m$

podemos calcular o raio orbital pós-excitação

$r=E_{0} t / 2 B$
Esta equação mostra que o raio de um ião excitado é independente de $m / q$, ou seja, iões de uma determinada gama de razões massa/carga podem ser excitados para um mesmo raio orbital de ciclotrão por aplicação de um campo eléctrico cuja intensidade seja constante com a frequência..Por exemplo, iões num campo magnético de $3 \mathrm{~T}$ são excitados para um raio de $1,67 \mathrm{~cm}$ por aplicação de um campo eléctrico de radiofrequências de intensidade constante de 1 $\mathrm{Vcm}^{-1}$, durante $1 \mathrm{~ms}$.

O movimento coerente dos iões resultante da excitação pode agora induzir um sinal eléctrico detectável nos pratos de recepção. Se estes estiverem ligados por um circuito de amplificação diferencial, será possível observar as pequenas correntes (designadas por "correntes-imagem") induzidas pelos "pacotes" de iões à medida que se movem sucessivamente para junto de um e outro dos pratos de detecção. Estas correntes oscilantes podem ser convertidas em voltagens, amplificadas e digitalizadas, dando origem a um sinal discreto no domínio do tempo (também designado por transiente) que é igual ao somatório das sinusóides representativas do movimento de ciclotrão de cada "pacote" de iões com uma mesma razão massa/carga. O sinal para cada grupo de iões tem a forma

$f(t) \propto N_{0} \exp (-t / \tau) \cos \left(\omega_{\mathrm{C}} t+\phi\right)$

em que $N_{0}, \tau$ e $\phi$ são, respectivamente, o número, a constante de amor- tecimento no domínio do tempo e a fase inicial dos iões com uma mesma razão $\mathrm{m} / q$.

A constante de amortecimento (ou tempo de relaxação) está presente na equação 18 pois o sinal de ICR só é detectado enquanto o "pacote" de iões mantiver um movimento coerente. O principal mecanismo de perda de coerência de fase dos iões é por colisão com as espécies neutras presentes na célula de ICR e, tal como se pode ver pela equação 18 , traduz-se num decréscimo exponencial do sinal transiente.

o sinal complexo no domínio do tempo pode, finalmente, ser descodificado por aplicação de uma transformação de Fourier, obtendo-se um sinal no domínio das frequências que contém a informação de frequências, fases e intensidades dos iões presentes na célula de ICR.

Aspectos experimentais. Um espectrómetro de massa FT-ICR actual é constituído, basicamente, por um sistema de alto vácuo capaz de produzir pressões de $10^{-9}$ torr, situado no centro de um campo magnético forte, habitualmente de 3 a $7 \mathrm{~T}$, gerado por um magnete supercondutor. No centro da câmara de vazio (e consequentemente do campo magnético) encontra-se a célula de ICR, geralmente de forma cúbica ou cilíndrica, embora outras geometrias, como por exemplo a hiperbólica ou variantes das formas cúbica e cilíndrica, sejam por vezes utilizadas em experiências com o objectivo de alte-

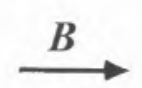

(a)

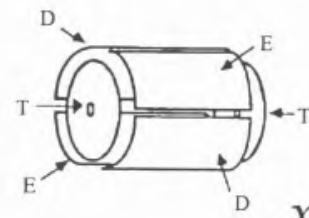

(b)

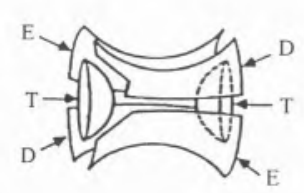

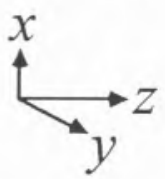

Fig. 4 - Representação esquemática de células de ICR com geometrias cilíndrica (a) e hiperbólica (b). E - excitação, D - detecção, T - confinamento ou "trapping". 


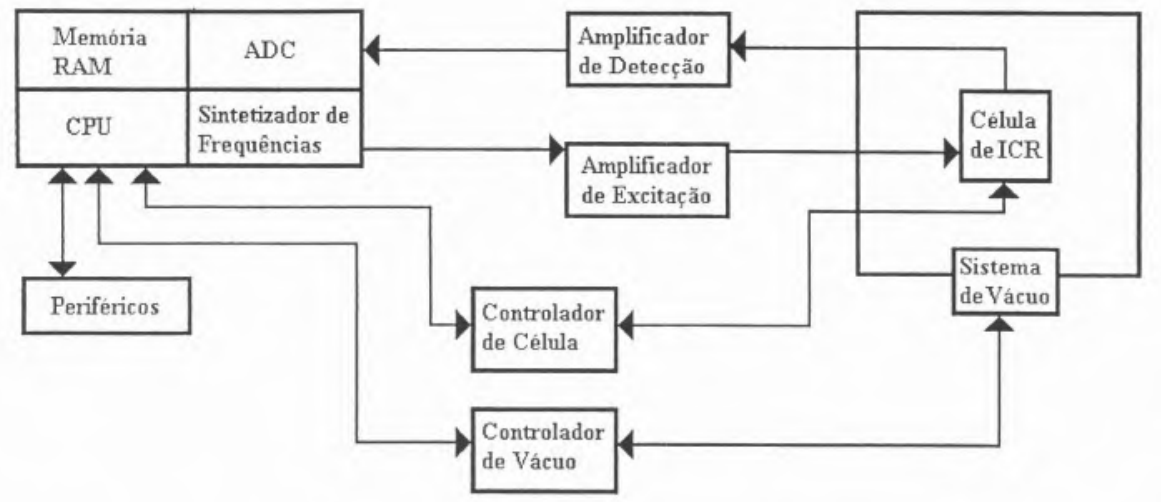

Fig. 5 - Diagrama de blocos das principais componentes de um espectrómetro de massa FT-ICR. rar e optimizar o campo eléctrico aplicado. Na Figura 4 encontram-se representadas células de ICR simples com as geometrias cilíndrica e hiperbólica; na Figura 1 foi já mostrada uma célula com forma cúbica. um espectrómetro de massa FT-ICR são as requeridas para efectuar o controle do instrumento, o processamento de sinal e o tratamento de dados. Na Figura 5 mostra-se um diagrama de blocos simplificado de um destes sistemas, que engloba controladores do sistema de vácuo (bombas lula de ICR (voltagens), um sintetizador de frequências, amplificadores de sinal de excitação e de detecção, um conversor analógico-digital (ADC), uma memória de acesso rápido (RAM), um processador central (CPU), sistemas de armazenamento de dados e periféricos.

Uma experiência de FT-ICR/MS é, na prática, uma sequência de eventos controlados por computador em que se manipulam os conjuntos de quanto que os vários passos de uma experiência realizada num dos espectrómetros de massa mais comuns (de sectores, de quadrupolo ou de tempode-voo) estão separados espacialmente, nas experiências de FT-ICR/MS essa separação é temporal. A Figura 6a mostra uma sequência de eventos elementar, constituída por passos (impulsos) de ionização, excitação, detecção e remoção ("quench"), este últi-
As componentes electrónicas de de vazio, válvulas e sondas) e da céiões confinados na célula de ICR. En- mo destinado a remover todos os iões presentes na célula de ICR, preparando-a para nova sequência. Este passo de remoção é habitualmente efectuado por aplicação de um potencial elevado, positivo e seguidamente negativo, aos pratos de confinamento, provocando a neutralização dos iões de sinal oposto ao do potencial aplicado. Adicionando diversas combinações de passos de excitação ou pausas (para colisão e reacção ião-molécula neutra), é possível construir sequências que correspondem a experiências de espectrometria de massa bastante elaboradas (MS/MS/...). Na Figura $6 \mathrm{~b}$ pode ver-se uma sequência experimental mais complexa, em que se utiliza a dissociação induzida por colisões (CID) para caracterizar um produto de uma reaç̧ão ião-molécula.

É possível estabelecer uma analogia entre uma experiência de FT-ICR/MS e as manipulações efectuadas num laboratório de química convencional, fazendo corresponder ao passo de ionização a síntese, ao de selecção de iões uma purificação, ao de colisão/reacção ião-molécula uma reacção química, aos passos de excitação/detecção a análise dos produtos da reacção e, finalmente, à remoção dos iões da célula de ICR a limpeza do material utilizado.

No passo inicial de formação de iões pode, em princípio, ser utilizado qualquer método de ionização actualmente em uso em espectrometria de massa. Teremos oportunidade de referir mais adiante os métodos de ionização mais usados em FT-ICR/MS, bem como as adaptações necessárias para que as experiências se tivessem tornado instrumentalmente realizáveis. No caso mais comum e simples do método de ionização por impacto electrónico (EI), a maioria dos espectrómetros FT-ICR possui um sistema constituído por filamento, rede aceleradora e colector capaz de produzir um feixe de electrões de alguns microamperes que atravessa a célula de ICR ao longo do eixo $z$, passando através de pequenos orifícios abertos no centro dos pratos de confinamento, e que vai criando iões no seu percurso no interior da célula.

Como foi referido, toda a manipulação dos iões na célula de ICR se baseia na utilização de diferentes combinações de pausas ("delays") e impulsos de excitação. A inclusão de pausas de duração variável nas sequências experimentais, durante as quais os iões podem reagir com espécies neutras presentes na câmara de vazio do espectrómetro de massa laí introduzidas através de válvulas de (a)

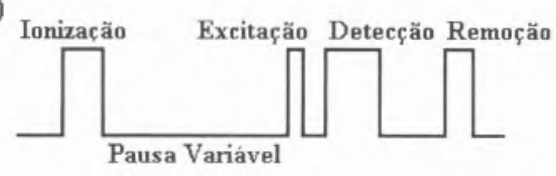

(b)

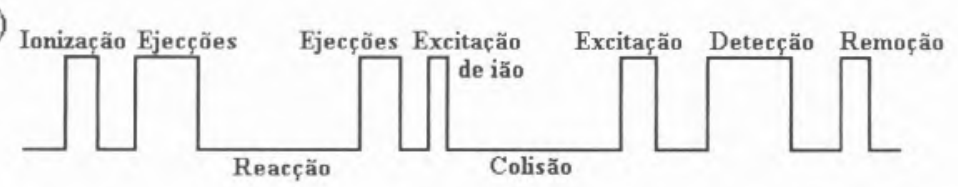

Fig. 6 - Representação esquemática de sequências experimentais de FT-ICR/MS. (a) Sequência de impulsos para experiência simples. (b) Sequência de impulsos para experiência de MS/MS. 
diferentes tipos), constitui a base da grande utilização da técnica de FT-ICR/MS no estudo de reacções ião-molécula em fase gasosa.

Os impulsos de excitação correspondem à aplicação de campos eléctricos de radiofrequências de diferentes formas, intensidades e durações aos pratos de excitação, que, como vimos na secção anterior, irão fornecer energia aos iões quando em ressonância, de acordo com a equação 15. Consoante o valor atingido pela energia cinética final dos iões, assim será possível por exemplo ejectar da célula de ICR um ou mais iões se o raio orbital final respectivo (ver equação 17) exceder as dimensões da célula. Este é o procedimento habitual para excluir iões indesejados de passos subsequentes de reacção, colisão ou da própria deteç̧ão; neste último caso, o objectivo pode ser por exemplo aumentar o intervalo dinâmico de análise ("dynamic range"). que em FT-ICR/MS é limitado a cerca de $10^{3}-10^{4}$. A utilização de ejecções selectivas de iões pode ser muito útil na elucidação de sequências de reacção ião-molécula e designa-se por ressonância dupla ("double resonance"), método que foi desenvolvido ainda na era dos espectrómetros ICR sem FT.

Um impulso de excitação pode também ser usado para fornecer a um ião energia translaccional suficiente para que, ao colidir com uma molécula neutra, sofra CID. As energias de colisão podem variar entre alguns $\mathrm{eV}$ e algumas centenas de eV, consideravelmente inferiores aos valores de alguns $\mathrm{keV}$ que se podem obter em espectrómetros de massa de sectores: além disso, após a excitação os iões possuem uma distribuição finita de energia cinética que conduz a uma menor reprodutibilidade dos espectros de CID obtidos por FT-ICR/MS. Por outro lado, a maior escala de tempos da espectrometria de massa FTICR origina a ocorrência de colisões múltiplas que podem favorecer processos de rearranjo dos iões. Uma das principais vantagens é a possibilidade de obtenção de espectros de alta resolução dos iões fragmento.
Os impulsos de excitação podem ter diferentes formas. Para além da irradiação numa frequência única que pode ser útil para ejectar da célula de ICR um dado ião ou para o excitar numa experiência de CID, é necessário produzir excitações em banda larga para, por exemplo, eliminar da célula iões de uma determinada gama de razões massa/carga e naturalmente para, no passo final de excitação de uma sequência experimental, preparar os iões presentes na célula para a detecção. Embora seja possível utilizar um impulso rectangular para excitar iões em banda larga, o método de excitação mais utilizado para este efeito é o varrimento de frequências ("chirp"), que produz uma excitação mais uniforme num dado intervalo de frequências e requer uma menor intensidade de campo eléctrico de excitação em comparação com o método de impulso.

Apesar destas vantagens, a excitação por varrimento de frequências não é completamente uniforme em todo o intervalo de frequências e apresenta "ombros" nos extremos do intervalo que the limitam a selectividade. O método de excitação desenvolvido por Marshall e colaboradores em meados dos anos 80 , designado por SWIFT ("Stored Waveform Inverse Fourier Transform"). apresenta características excepcionais de uniformidade e selectividade de excitação e/ou ejecção, que resultam da forma como é construído o impulso de excitação. O facto de a transformada de Fourier funcionar tanto em sentido directo (do domínio do tempo para o das frequências) como em sentido inverso (do domínio das frequências para o do tempo), permite que se possa desenhar um espectro de frequências correspondente a um perfil de excitação desejado e, através de uma transformada de Fourier inversa, produzir uma onda de excitação no domínio do tempo que, num único impulso, pode efectuar combinaçōes variadas de excitações e/ou ejecçōes.

O passo final de uma sequência experimental de FT-ICR/MS é cons- tituído pela detecção, a que se segue naturalmente o tratamento do sinal transiente obtido até chegar ao espectro de massa. A espectrometria FT-ICR é a única técnica de análise de massas que detecta os iões não destrutivamente, pois, como vimos anteriormente, utiliza as correntes imagem induzidas nos pratos de detecção da célula de ICR pelos "pacotes" de iões a moverem-se em fase. O sinal transiente é uma sobreposição das frequências de ciclotrão dos iões excitados, que é depois convertido numa voltagem, digitalizado e sujeito a uma transformada de Fourier que revela o espectro das frequências. Como em outras técnicas instrumentais, podem acumular-se vários sinais transientes para melhorar a razão sinal/ruído.

Uma vez que é possível medir frequências com uma exactidão extremamente elevada (1 parte em 109), a técnica tem a capacidade de efectuar mediçōes de massas com exactidão ultra-elevada, desde que seja utilizada uma lei de calibração de massas adequada. Como as frequências detectadas correspondem às frequências de ciclotrão reduzidas, a relação massa/frequência rege-se pela equação 9 , da qual é possível deduzir a seguinte equação de calibração, correntemente usada em FT-ICR/MS

$$
m / q=A / v_{\mathrm{R}}+B / v_{\mathrm{R}}^{2}
$$

$A$ e $B$ são constantes que podem ser obtidas a partir das frequências dos picos de pelo menos dois iões com razões $m / q$ conhecidas. A grande estabilidade dos campos magnéticos produzidos pelos magnetes supercondutores actualmente usados possibilita a utilização de uma mesma calibração durante períodos de tempo relativamente longos.

Outra das características mais importantes da espectrometria de massa FT-ICR é o seu elevado poder de resolução de massas. A partir da equação fundamental de ciclotrão, equação 3, pode obter-se, por derivação em ordem a $m$ e para uma gama de massas ou frequências suficientemente pequena, a seguinte relação 


$$
\omega / \Delta \omega=-m / \Delta m
$$

que mostra que em FT-ICR a resolução de frequências é equivalente à resolução de massas. $\Delta \omega$ é habitualmente definida como a largura de um pico a meia altura no espectro no domínio das frequências. Como a frequência é dada aproximadamente pela equação de ciclotrão, o poder de resolução de massas pode ser expresso como

$$
m / \Delta m=-q B / m \Delta \omega
$$

ou seja, é inversamente proporcional à massa e directamente proporcional à intensidade do campo magnético. $\mathrm{O}$ poder de resolução de massas aumenta também proporcionalmente com o tempo de observação; como a duração de um sinal transiente depende essencialmente da pressão na célula de ICR, pois as colisões ião-molécula provocam a perda de coerência do movimento dos iões, um aumento na pressão de trabalho origina uma diminuição da resolução. Para uma pressão de cerca de $10^{-8}$ torr, pode obter-se, com relativa facilidade, um poder de resolução de $10^{6}$ para uma massa 100 na maioria dos espectrómetros FT-ICR comerciais. Também a razão sinal/ruído aumenta com o tempo de observação e assim, resolução e sensibilidade podem aumentar simultaneamente, facto que constitui uma característica única da espectrometria de massa FT-ICR, pois nos espectrómetros de massa mais comuns um aumento de resolução tem como consequência uma diminuição de sensibilidade. É também possível aumentar o tempo de observação simplesmente aumentando a memória do computador, o que corresponde à existência de um maior número de pontos disponíveis para a conversão analógicodigital de um sinal.

O poder de resolução e a exactidão na determinação das massas podem ter uma limitação no número de iões presentes na célula de ICR. Se este número for muito grande, superior a $10^{5}$ para um campo magnético de $3 \mathrm{~T}$, torna-se necessário considerar os efeitos das repulsões ião-ião (efeitos de carga espacial). Uma vez que o campo electromagné- tico estabelecido numa célula de ICR típica não é completamente uniforme, as repulsões de Coulomb podem mover alguns iões para regiões do espaço em que o campo aplicado é ligeiramente diferente e assim desviar, alargar e distorcer os picos de um espectro de massa.

Foi referido anteriormente que existe uma massa crítica, $m_{\mathrm{c}}$, acima da qual a trajectória de um ião na célula de ICR deixa de ser estável, massa essa dada pela equação 14. Para alargar o limite superior de detecção da técnica pode utilizar-se, por exemplo, e de acordo com a referida equação. um campo magnético mais elevado $\left(m_{\mathrm{c}}\right.$ é directamente proporcional a $B^{2}$ ), uma célula de maiores dimensões $\left(m_{\mathrm{c}}\right.$ é directamente proporcional a $a^{2}$, um menor potencial de confinamento $\left(m_{\mathrm{c}}\right.$ é inversamente proporcional a $V_{\mathrm{T}}$ ) ou uma diferente geometria da célula ( $m_{\mathrm{c}}$ é inversamente proporcional a $\alpha$ ). Além desta limitação proveniente da existência de uma massa crítica, a detecção de massas muito elevadas, a que correspondem frequências bastante baixas, pode sofrer interferências importantes do ruído de fundo ambiente. Na prática, podem introduzir-se melhoramentos na electrónica para reduzir essas interferências ou utilizar-se um campo magnético mais intenso, que fará corresponder a uma mesma razão $\mathrm{m} / \mathrm{q}$ uma frequência mais elevada.

O limite inferior do intervalo de massas detectável é determinado pela velocidade do digitalizador de sinal, que deve ser igual ao dobro da frequência observável mais elevada pois, de acordo com o teorema de amostragem de Nyquist, para conhecer a verdadeira frequência de uma sinusóide é necessário proceder a uma amostragem pelo menos duas vezes por ciclo. Se for utilizado um digitalizador de 5,2 MHz, a frequência mais elevada que pode ser processada sem originar distorções é de 2,6 $\mathrm{MHz}$ que, para uma intensidade de campo magnético de $3 \mathrm{~T}$, corresponde a uma massa de cerca de 18 Da. Para detectar massas mais baixas será necessário utilizar um digitalizador mais rápido ou um campo magnético menos intenso. O teorema de Nyquist explica também o fenómeno de aparecimento num espectro de picos "dobrados" ("folded back", fenómeno de "foldover"), que correspondem a iões excitados cujas massas se encontram fora do intervalo de detecção. Para uma dada velocidade de digitalização do sinal, frequências que excedam a largura de banda de observação podem surgir como frequências mais baixas, pois a amostragem do sinal analógico não é suficientemente rápida. Este fenómeno pode ser aproveitado para detectar iões cuja massa seja menor que o limite inferior do intervalo de deteç̧ão, num determinado instrumento.

Temos vindo a referir o modo de detecção que se designa por directo ou de banda larga, pois o sinal que é digitalizado e transformado é obtido directamente a partir da voltagem diferencial induzida entre os dois pratos de detecção. É possível usar um outro modo de deteç̧ão, denominado "heterodyne" ou de banda estreita, que permite obter espectros de resolução ultra-elevada. Na detecção "heterodyne" o sinal proveniente dos pratos de detecção é misturado com um sinal de referência com uma frequência próxima das que correspondem aos iões a ser observados, mistura essa que produz dois novos sinais com frequências iguais à soma e à diferença respectivas; o sinal soma é eliminado por um filtro e apenas o sinal diferença é tratado, conseguindo-se assim desviar um dado intervalo de frequências para valores mais baixos, o que possibilita a utilização de maiores tempos de aquisição de sinal, com um consequente aumento da resolução,

A existência de não linearidades na experiência de FT-ICR pode levar ao aparecimento de sinais que não correspondem apenas às três frequências naturais de movimento dos iões, e podem surgir nos espectros de massa picos correspondentes a frequências múltiplas da 'fundamental (harmónicas) ou a combinações das frequências naturais (modulação que origina bandas laterais, "sidebands"). Um exemplo do primeiro tipo de sinal 
pode surgir quando o raio orbital de um ião após a excitação se encontra muito perto dos pratos de deteç̧ão, dando origem a um sinal transiente que não é mais uma sinusóide mas se aproxima de uma onda quadrada, cujo espectro contém picos na frequência fundamental e nas harmónicas ímpares. O segundo tipo de sinal resulta do facto de os iões descreverem uma combinação dos movimentos de ciclotrão reduzido e de oscilação axial, podendo surgir picos-satélite ("sidebands") a frequências $v_{\mathrm{R}} \pm$ $2 v_{\mathrm{T}}$. Na prática, todos estes sinais têm geralmente uma intensidade bastante baixa relativamente à da frequência fundamental de ciclotrão.

Uma das principais vantagens da espectrometria de massa FT-ICR resulta da utilização da transformação de Fourier. Tal como acontece com outras espectroscopias que empregam este tipo de operação matemática, a deteç̧ão simultânea da totalidade de um espectro, a partir de um sinal no domínio do tempo, tem uma vantagem, denominada multicanal ou de Fellgett, que se traduz, para um espectro com $N$ pontos, por um ganho em tempo, para uma mesma razão sinal/ruído, de um factor $N$, ou um ganho em razão sinal/ruído, para um dado tempo de aquisição de dados, de um factor $N^{1 / 2}$, quando comparada com uma deteç̧ão efectuada por varrimento dos elementos espectrais um a um.

Diversos procedimentos comuns às espectroscopias FT são habitualmente usados em FT-ICR/MS, de que é exemplo a apodização. Neste procedimento, o sinal no domínio do tempo é multiplicado por uma função matemática de tipo exponencial que, após a transformação de Fourier, pode conduzir a um espectro com melhor razão sinal/ruído ou resolução. A apodização é também uma maneira efectiva de remover o alargamento da base dos picos que resulta da respectiva forma Lorentziana, e que a existir, pode obscurecer a presença de picos vizinhos de baixa intensidade.

A utilização de baixas pressões é um requisito essencial para que a espectrometria de massa FT-ICR possa realizar todas as suas capacidades analíticas. A configuração original dos instrumentos de FT-ICR/MS com uma célula de ICR única colocada no interior de um sistema de vazio simples revelou-se incompatível com alguns dos métodos de ionização habitualmente usados em espectrometria de massa, como por exemplo o bombardeamento com átomos rápidos (FAB), ou para a utilização das chamadas técnicas hifenadas, como por exemplo para o acoplamento de cromatografia gasosa (GC). Em consequência deste facto, foram desenvolvidos espectrómetros em que se utilizam bombagens diferenciadas da zona de entrada de amostras e/ou de ionização e da zona de análise. Na Figura 7 encontram-se esquematizados os dois tipos principais de configurações desenvolvidas e correntemente usadas: a configuração com fonte externa (Figuras 7a e 7b) e a configuração com célula de ICR dupla (Figura 7c).

Nos instrumentos com fonte externa, os iōes são criados exteriormente ao campo magnético e transferidos para a célula de ICR através de sistemas de multipolos (Figura 7a) ou de lentes electrostáticas (Figura $7 \mathrm{~b}$ ). Normalmente utilizam-se três estádios de bombagem diferencial para reduzir a pressão da gama dos militorr na zona da fonte de ionização para pressões inferiores a 108 torr na região da célula de ICR. Os aparelhos com célula dupla (Figura 7c) são constituídos por duas câmaras de vazio, com bombagem independente, em cujo centro se encontram as duas células que partilham o prato de confinamento central. As câmaras de vazio comunicam entre si por um limite de condutância, ou seja, um pequeno orifício que permite manter entre as duas regiões uma razão de pressões de $10^{2}-10^{3}$. Os iões podem ser transferidos de uma célula para a outra levando a zero o potencial do prato de confinamento central durante tempos determinados, constituindo apenas mais um passo nas sequências experimentais. A configuração com fonte externa é, potencialmente, a de maior utilidade pois permite, em princípio, adaptar qualquer tipo de fonte de ionização ao analisador de massas FT-ICR.

A finalizar esta secção, em que se apresentaram diversos aspectos práticos da espectrometria de massa FT-ICR, referiremos um dos seus desenvolvimentos instrumentais mais recentes, que recebeu a designação de axialização quadrupolar. Trata-se de uma técnica de excitação que utiliza os quatro pratos da célula de ICR que são paralelos ao eixo do campo magnético (pratos de excitação e de deteç̧ão) para, por conversão do movimento de magnetrão dos iões em movimento de ciclotrão na presença de colisões ião-neutro, obrigar uma nuvem de iões dispersos a formarem um "pacote" compacto de iões no centro da célula. A axialização quadrupolar pode, em princípio, melhorar todos os aspectos de uma experiência de FT-ICR, designadamente o poder de resolução de massas, a exactidão, a razão sinal/ruído, a eficiência de medição repetida dos mesmos iões, a selectividade das experiências de MS/MS e a eficiência de confinamento na presença de pressōes elevadas de neutros.

\section{FT-ICR/MS: APLICACÕES}

As principais aplicações da espectrometria de massa FT-ICR em química podem agrupar-se em duas grandes áreas, a saber, a química-física, em particular no domínio da química de iões em fase gasosa, e a química analítica, em especial na caracterização de moléculas de massa elevada.

A espectrometria de massa FT-ICR tem demonstrado ser uma técnica de eleição para o estudo de reacções ião-molécula em fase gasosa devido à sua capacidade para confinar e manipular os iões. Com estes estudos tem sido possível obter informação de natureza cinética, termoquímica e mecanística em inúmeros sistemas orgânicos e organometálicos, fazendo uso frequente de técnicas como CID e fotodissociação; no caso dos sistemas envolvendo iões metálicos, a compatibilidade da técnica com a ionização com laser (LI) veio facilitar a 
obtenção de iões metálicos para estudos de reactividade com substratos orgânicos. Os estudos de química de iões em fase gasosa por FT-ICR/MS têm-se revelado como um complemento importante dos estudos desenvolvidos nesta área através de outras técnicas de espectrometria de massa.

$\mathrm{O}$ interesse analítico da espectrometria de massa FT-ICR provém fundamentalmente da reunião numa única técnica de um elevado poder de resolução de massas, de uma elevada exactidão na sua medição e da capacidade de estudar a estrutura dos iões através de diversas técnicas de dissociação. O grande interesse actual da técnica é resultante da demonstração, após o desenvolvimento dos espectrómetros FT-ICR com fonte externa de iões, da sua compatibilidade com os métodos de ionização mais utilizados nos últimos anos, em particular a dessorção/ionização com laser assistida por matriz (MALDI) e a ionização por electrovaporização (ESI). Embora sejam variadas as aplicações da técnica, por exemplo, na análise de superfícies e de polímeros, tem sido na caracterização de biomoléculas de massa elevada, designadamente peptídeos, proteínas, oligonucleotídeos e oligossacarídeos, que a espectrometria de massa FT-ICR tem encontrado aplicação privilegiada nos últimos anos, acompanhando a utilização crescente da espectrometria de massa na investigação em bioquímica.

Na bibliografia indicam-se vários artigos de revisão que ilustram em pormenor a grande diversidade das aplicações da espectrometria de massa FT-ICR nas referidas áreas da química de iões em fase gasosa e da química analítica.

\section{CONCLUSÃO}

Os primeiros espectrómetros de massa FT-ICR comerciais surgiram em 1981 e desde então tem-se observado um crescimento constante do número de aparelhos em utilização em todo o mundo, atingindo hoje um número superior a 200. Em Por-

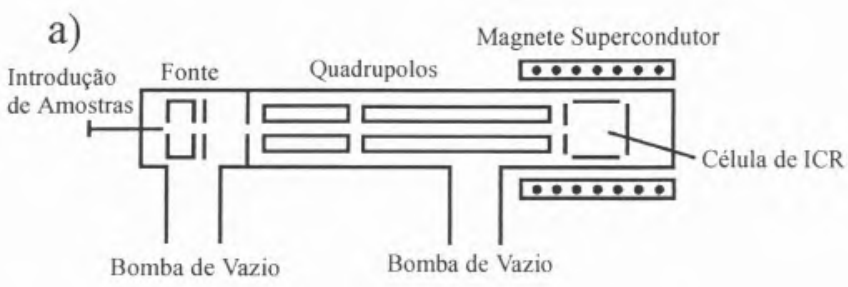

b)

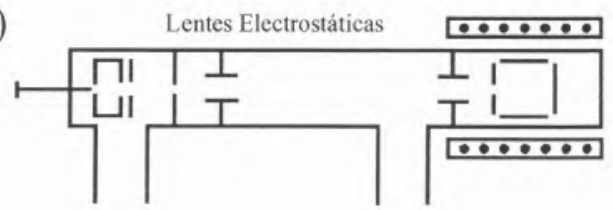

c)

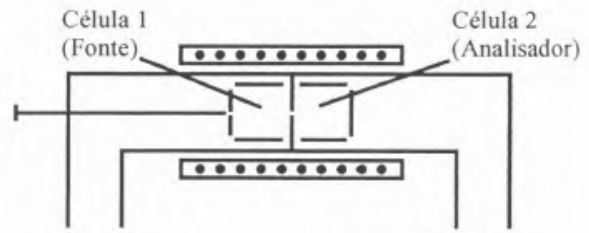

Fig. 7 - Representação esquemática de espectrómetros de massa FT-ICR. (a) Configuração com fonte externa e multipolos. (b) Configuração com fonte externa e lentes electrostáticas. (c) Configuração com célula de ICR dupla.

tugal, encontra-se em operação há cerca de 6 anos um espectrómetro de massa FT-ICR, adquirido no âmbito do Programa "Ciência" por proposta de grupos de investigação do Instituto Tecnológico e Nuclear, da Faculdade de Ciências da Universidade de Lisboa e do Instituto Superior Técnico. O espectrómetro, com uma configuração com célula de ICR dupla e dispondo de ionização com laser, está instalado no Departamento de Química do ITN, em Sacavém, e tem sido utilizado principalmente em estudos de química de iões em fase gasosa, embora com uma contribuição apreciável na análise de compostos $\mathrm{e}$ materiais de diversos tipos.

Nos últimos anos tem-se observado um desenvolvimento assinalável da espectrometria de massa FT-ICR que, estimulado pelo seu uso crescente em aplicações no âmbito da bioquímica, se prevê que continue nos próximos anos. A nível instrumental poderá esperar-se que a consolidação e aperfeiçoamento de diversos aspectos particulares como o desenho de células de ICR para optimização do campo eléctrico, a transferên- cia de iões das fontes externas para a zona de análise, o uso crescente da axialização quadrupolar e de novas técnicas de dissociação como SORI ("sustained off-resonance irradiation"), MECA ("multiple excitation collisional activation") e BIRD ("blackbody infrared radiative dissociation"), o desenvolvimento de sequências especiais como SWIM ("stored waveform ion modulation" ou 2D-FT-ICR) e ainda a utilização de magnetes com campos mais elevados e o aproveitamento dos melhoramentos constantes dos computadores, tenham implicações relevantes nas capacidades da técnica, expandindo as suas aplicações analíticas e também a sua utilização ao nível da química de iões em fase gasosa.

\section{AGRADECIMENTOS}

Agradeço aos Doutores António Pires de Matos e João Paulo Leal, do ITN, a leitura atenta do manuscrito.

\footnotetext{
* Departamento de Química, Instituto Tecnológico e Nuclear, Estrada Nacional 10 2686 Sacavém Codex
} 


\section{BIBLIOGRAFIA}

A. G. Marshall, F. R. Verdun, Fourier Transforms in NMR, Optical, and Mass Spectrometry - A User's Handbook, Elsevier, Amsterdam, 1990

A. G. Marshall, M. B. Comisarow, "Fourier and Hadamard Transform Methods in Spectroscopy", Anal. Chem., 47 (1975) 491A.

L. Glasser, "Fourier Transforms for Chemists", J. Chem. Educ. 64 (1987) A228 (Parte I), A260 (Parte II), A306 (Parte III).

A. G. Marshall, "Fourier Transform Ion Cyclotron Resonance Mass Spectrometry", Acc. Chem. Res., 18 (1985) 316.

A. G, Marshall, P. B. Grosshans, "Fourier Transform Ion Cyclotron Resonance Mass Spectrometry: the Teenage Years", Anal. Chem., 63 (1991) 215A.

A. G. Marshall, "Ion Cyclotron Resonance and Nuclear Magnetic Resonance Spectroscopies: Magnetic Partners for Elucidation of Molecular Structure and Reactivity", Acc. Chem. Res., 29 (1996) 307.

S. Guan, H. S. Kim, A. G. Marshall, M. C. Wahl, T. D. Wood, X. Xiang, "Shrink-Wrapping an lon Cloud for HighPerformance Fourier Transform Ion Cyclotron Resonance Mass Spectrometry", Chem. Rev., 94 (1994) 2161.

S. Guan, A. G. Marshall, "Ion Traps for Fourier Transform Ion Cyclotron Resonance Mass Spectrometry: Principles and Design of Geometric and Electric Configurations", Int. I. Mass Spectrom. Ion Processes, 146/147 (1995) 261.
S. Guan, A. G. Marshall, "Stored Waveform Inverse Fourier Transform (SWIFT) Ion Excitation in Trapped-lon Mass Spectrometry: Theory and Applications", Int. I. Mass Spectrom. Ion Processes, 157/158 (1996) 5 .

A. G. Marshall, S. Guan, "Advantages of High Magnetic Field for Fourier Transform Ion Cyclotron Resonance Mass Spectrometry", Rapid Commun. Mass Spectrom., 10 (1996) 1819.

M. V. Buchanan (Editor), Fourier Transform Mass Spectrometry: Evolution, Innovation, and Applications, American Chemical Society, Washington, DC, 1987.

B. S. Freiser, "Fourier Transform Mass Spectrometry", em I. M. Farrar, W. H. Saunders (Editores), Techniques for the Study of lon-Molecule Reactions, Wiley, New York, 1988. Capítulo 2

V. H. Vartanian, I. S. Anderson, D. A. Laude "Advances in Trapped Ion Cells for Fourier Transform Ion Cyclotron Resonance Mass Spectrometry", Mass Spectrom. Rev., 14 (1995) 1.

C. L. Holliman, D. L. Rempel, M. L. Gross, "Detection of High Mass-to-Charge lons by Fourier Transform Mass Spectrometry", Mass Spectrom. Rev., 13 (1994) 105.

M. V. Buchanan, R. L. Hettich, "Fourier Transform Mass Spectrometry of High-Mass Biomolecules", Anal. Chem., 65 (1993) $245 \mathrm{~A}$

T. Dienes, S. I Pastor, S. Schürch, 1, R. Scott, I, Yao, S Cui, C. L. Wilkins, "Fourier Transform Mass Spectrometry - Ad- vancing Years (1992-mid. 1996)", Mass. Spectrom, Rev, 15 (1996) 163.

M. T. Bowers, A. G. Marshall, F. W. McLafferty, "Mass Spec trometry: Recent Advances and Future Directions", I. Phys. Chem., 100 (1996) 12897.

N. M. M. Nibbering, "Gas-Phase lon/Molecule Reactions as Studied by Fourier Transform Ion Cyclotron Resonance", Acc. Chem. Res., 23 (1990) 279.

H. I. Kenttämaa, "Long-lived Distonic Radical Cations", Org. Mass Spectrom., 29 (1994) 1.

K. Eller, H. Schwarz, "Organometallic Chemistry in the Gas Phase", Chem. Rev., 91 (1991) 1121.

B. S. Freiser, "Selected Topics in Organometallic lon Che mistry", Acc. Chem. Res., 27 (1994) 353.

B. S. Freiser, "Methods for Determining Metal-Ligand and Metal-Metal Bond Energies Using Fourier Transform Mass Spectrometry", em T. I. Marks (Editor), Bonding Energetics in Organometallic Compounds, American Chemical Society, Washington, DC, 1990, Capítulo 4.

J. A. Martinho Simōes, J. L. Beauchamp, "Transition MetalHydrogen and Metal-Carbon Bond Strengths: The Keys to Catalysis", Chem. Rev., 90 (1990) 629.

P. Sharpe, D. E. Richardson, "Applications of Fourie Transform lon Cyclotron Resonance Mass Spectrometry in Coordination Chemistry", Coord. Chem. Rev. 93 (1989) 59.

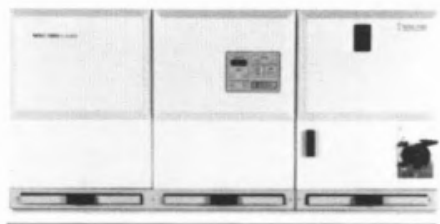

SPECTROFLAME MODULA

ICP para análise simultânea e/ou sequencial de elementos quimicos em solucăo

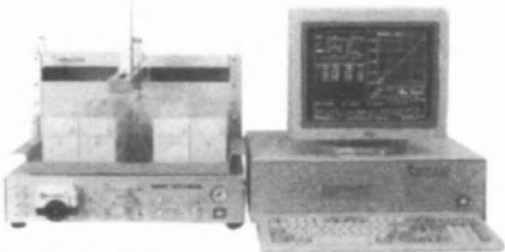

\section{SPECTROMERC}

Analisador de mercurio para operaçào manual automática ou acoplado ao ICP

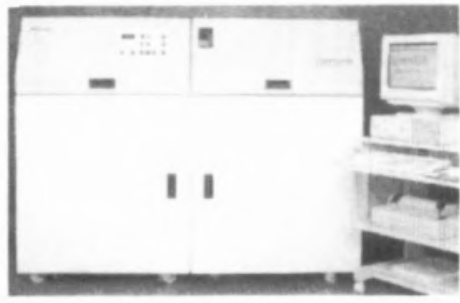

SPECTROMASS - ICP

Espectrómetro de massa - ICP para análise de vestigios de elementos e isótopos

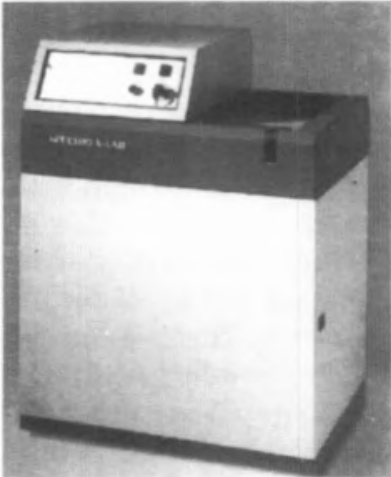

X-RAY

Espectrometro de fluorescência de energia dispersiva de Raio-X para determinaçăo de elementos quimicos em amostras solidas e liquidas 